\title{
3D Pose Refinement Using Rendering and Texture-Based Matching
}

\author{
Xenophon Zabulis ${ }^{1}$, Manolis I.A. Lourakis ${ }^{1}$, and Stefanos S. Stefanou ${ }^{2}$ \\ ${ }^{1}$ Institute of Computer Science, FORTH \\ Nikolaou Plastira 100, GR - 70013, Heraklion, Greece \\ 2 Institute of Molecular Biology and Biotechnology, FORTH \\ Nikolaou Plastira 100, GR - 70013, Heraklion, Greece \\ \{zabulis,lourakis\}@ics.forth.gr, stefanou@imbb.forth.gr
}

\begin{abstract}
This paper presents a method for accurately determining the pose of Lambertian rigid objects present in an image. An initial pose estimate computed with the aid of local point features is ameliorated by considering all visible object texture. This is achieved by combining a textured mesh model of the object with a graphics renderer to synthesize an image of the object as would be captured by the camera at a particular pose. A rendered image is compared against the acquired one with the aid of a visual dissimilarity score involving cross-correlation. Populationbased stochastic optimization is used to efficiently search the pose space and minimize the dissimilarity between rendered images corresponding to candidate poses and the acquired image. The method is demonstrated with the aid of real and synthetic images.
\end{abstract}

\section{Introduction}

Object pose estimation refers to determining the six degrees of freedom defining the position and orientation of a rigid object relative to a camera. Typically, a 3D model is used to provide pose estimation with geometric knowledge about the object of interest. Such model-based approaches are usually preferred due to their increased robustness and fail-safety [10.

This work is concerned with the refinement of an initial pose estimate in order to improve its accuracy. This initial estimate is determined by extracting natural features from an image, matching them to an object model constructed offline and, finally, using the 3D coordinates of matched model points to estimate the 3D pose of the object relative to the camera. Refinement of the initial estimate is based on matching all observed object pixels against synthetic images of a textured triangle mesh model of the object. Derivative-free particle swarm optimization is used to explore the pose space with a population of candidate poses, without resorting to exhaustive search. Each candidate pose is evaluated by generating a synthetic image which predicts the appearance of the model at that pose and then measuring the photoconsistency between the synthetic image and the acquired one. The proposed approach employs a fully projective imaging formulation, improves the accuracy of feature-based pose estimates and 
can serve as the basis for drift-free tracking. Furthermore, it does not require any image preprocessing (e.g. no edges or silhouette need to be extracted) and employs all available texture information.

\section{Related Work}

Using features to estimate pose originated with [13]. Subsequent developments in covariant detectors and descriptors for image patches enabled the construction of compact object representations. For example, geometric models are combined with feature matching in 22 to track rigid objects in 3D. In [19, the appearance of object surface patches is captured using affine invariant local descriptors and their spatial relationships using multi-view geometric constraints. In 8 , a system is described based on SIFT features for recognizing learnt models in new images and solving for their pose. By relying on randomized trees to learn both appearance and geometry during a training phase, [15] circumvents the need to construct a prior 3D model. Similar to our feature-based pose estimation is the work in 18, that describes a system based on natural features.

Often, pose estimation with sparse features suffers from inaccuracies due to the limited number of correspondences. This boils down to the substantial differences in viewpoints among the images used to build the model and those used to estimate its pose. Feature-based pose estimates can be refined with the aim of increasing their accuracy, relying on information provided by pixels inside the object's projection in the form of optical flow, matched templates or edges. Relevant existing approaches are briefly reviewed next; a more detailed review can be found in [10. In 2, the object contour is combined with optical flow. However, it is assumed that the initial pose is known accurately whereas tracking failures cannot be recovered from. In [3], dense optical flow is complemented with tracked SIFT features. Wide-baseline stereo tracking is performed in 6 by augmenting the object contour with local descriptors of interest points. The idea of analysis by synthesis is also used in our approach, however we do not need to extract point features or contours from the rendered images. Reference [7] extends [6] to track articulated objects in the presence of self-occlusions, multiple moving objects and clutter using a combination of patch-based and region-based matching. The PWP3D algorithm in [17] adopts a probabilistic framework for simultaneous segmentation and pose tracking. PDFs are used to model image regions in [20, without needing to explicitly estimate contours in images. Among the reviewed methods, only [6]7] use rendering-based synthesis.

\section{Proposed Method}

\subsection{Model Acquisition}

The object model is comprised of two view-independent parts, namely a textured $3 \mathrm{D}$ mesh and a set of sparse 3D points upon its surface. To obtain a complete mesh, several images depicting the object from multiple unknown viewpoints 
are provided to a multiview, dense stereo reconstruction system 23. The model is represented by a textured mesh of triangles, let $M$, which is comprised by an ordered set of 3D points $V$ and an ordered set $T$ of triplets of indices on $V$ that determine the triangles of the mesh. A composite image $I$ collecting pixels from the images used in the reconstruction, is used to texture the $3 \mathrm{D}$ model. An ordered set $C$ of $2 \mathrm{D}$ points provides the texture coordinates in $I$ for each point from $V$.

Features from the surface of the object are also recovered. The images used to obtain the mesh are employed in a feature-based, structure-from-motion pipeline to recover a 3D point cloud 21. These 3D points may not exactly occur on the surface of the mesh due to inaccuracies between the two reconstructions. The $3 \mathrm{D}$ coordinates of the reconstructed points are associated with a SIFT feature descriptor [14]. A descriptor may be available from each image where a particular 3D point is imaged. We select the descriptor from the image in which the imaged surface is close enough and most frontal to the camera, guided by the surface normal as availed from the closest triangle in $M$. The true scale of the model is estimated as explained in [11].

\subsection{Pose Initialization}

The monocular pose estimation approach employed for initializing pose is outlined next and described in more detail in [12. First, SIFT keypoints are extracted from the image region occupied by the object and matched against those of its model to establish putative 2D-3D point correspondences. A P3P solver embedded into a RANSAC [5] framework estimates a preliminary pose estimate along with a classification of correspondences into inliers and outliers. The pose computed by RANSAC is next refined to take into account all inlying correspondences by minimizing a non-linear cost function amounting to their total reprojection error. Denoting by $\mathbf{K}$ the $3 \times 3$ intrinsic calibration matrix and corresponding 2D-3D points by $\mathbf{m}_{i}$ and $\mathbf{M}_{i}$, the cumulative image reprojection error is minimized with

$$
\min _{\mathbf{r}, \mathbf{t}} \sum_{i=1}^{n} d\left(\mathbf{K} \cdot[\mathbf{R}(\mathbf{r}) \mid \mathbf{t}] \cdot \mathbf{M}_{i}, \mathbf{m}_{i}\right)^{2}
$$

where $\mathbf{t}$ and $\mathbf{R}(\mathbf{r})$ are respectively the sought translation and rotation matrix parameterized using the Rodrigues rotation vector $\mathbf{r}, \mathbf{K} \cdot[\mathbf{R}(\mathbf{r}) \mid \mathbf{t}] \cdot \mathbf{M}_{i}$ is the predicted projection on the image of the homogeneous point $\mathbf{M}_{i}$ and $d(\mathbf{x}, \mathbf{y})$ denotes the reprojection error, i.e. the distance between points $\mathbf{x}$ and $\mathbf{y}$.

\subsection{Rendering Pose Hypotheses}

During evaluation of candidate poses, the model is rendered accordingly in synthetic images. The simulated virtual camera shares the same intrinsic and extrinsic parameters with the actual one, as were obtained via grid-based calibration. 

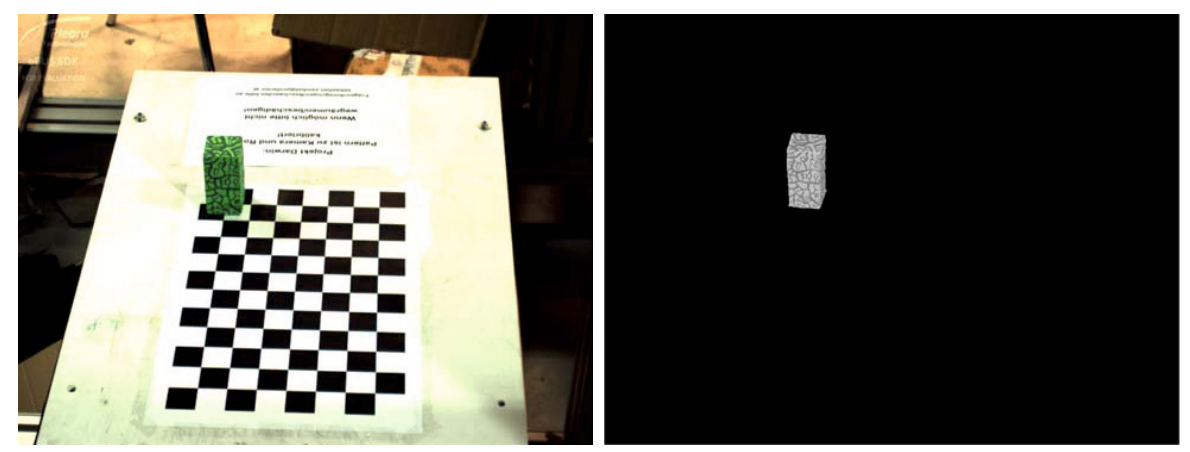

Fig. 1. Rendering pose hypotheses. Original image from an experiment (left) and a synthetic image generated for the evaluation of a pose hypothesis (right).

It is assumed that the 3D model $M$ is already transformed by the initial pose estimate (see Sec. 3.2), which is to be refined. Let $\mathbf{R}_{i}, \mathbf{t}_{i}$ be the $i$-th candidate pose for which the synthetic image $S_{i}$ is rendered. $M$ is transformed according to $\mathbf{R}_{i}, \mathbf{t}_{i}$ and $S_{i}$ is rendered as follows. Transformation $\mathbf{R}_{i}$, is an "in place" rotation. In other words, denoting by $\mathbf{c}$ the centroid of the points in $V$, the model is first translated by $-\mathbf{c}$, then rotated by $\mathbf{R}_{i}$, and finally translated back in place by c. Translation $\mathbf{t}_{i}$ is applied and the resulting model is rendered. Thus, model point $\mathbf{x}$ is transformed as $\mathbf{R}_{i} \cdot(\mathbf{x}-\mathbf{c})+\mathbf{c}+\mathbf{t}_{i}$. Taking also into account the transform, let $\mathbf{R}_{0}, \mathbf{t}_{0}$, availed from pose initialization (cf. Sec. 3.2), the overall transformation is $\mathbf{R}_{i} \cdot \mathbf{R}_{0} \cdot \mathbf{x}+\mathbf{R}_{i} \cdot\left(\mathbf{t}_{0}-\mathbf{c}\right)+\mathbf{c}+\mathbf{t}_{i}$.

Rendering of the synthetic image is carried out on the GPU and is implemented through OpenGL calls. The process employs Z-buffering to respect visibility and thereby project the $3 \mathrm{D}$ model realistically to the image plane.

\subsection{Evaluating Pose Hypotheses}

The accuracy of a candidate pose is evaluated with respect to its similarity to the acquired image. Normalized Cross Correlation (NCC) is used as a metric to quantify this similarity. Similarity is assessed pixel-wise and in the intensity domain. No segmentation procedure is involved in this process; thus for inaccurate poses, the rendered object is compared against pixels of the background.

The rendering process depicts the object against a neutral background and provides a mask indicating the foreground pixels. NCC is computed only for these pixels. Each foreground pixel is corresponded with the pixel of the acquired image at the same coordinates. The two pixel sets are collected with their elements being in a 1-1 correspondence. This is illustrated in Fig. 1 pixels rendered in the right image are being compared to the corresponding ones in the left. The NCC score is obtained from these pixel sets and is in $[-1,1]$, with -1 and 1 amounting to no similarity and maximum similarity, respectively. 


\subsection{Pose Refinement}

Pose is refined by searching the space around the initial estimate obtained in Sec. 3.2. Searching this space exhaustively incurs high computational costs, therefore a numerical optimization approach is adopted. The objective function is the similarity between the rendered and the imaged objects. The domain of optimization is restricted to a $6 \mathrm{D}$ neighborhood around the initial pose estimate. The objective function $\mathcal{O}$ is defined as the opposite of the NCC score.

The objective function $\mathcal{O}$ exhibits local minima [17. Thus, local optimization methods (e.g. gradient descent, Gauss-Newton or Levenberg-Marquardt) assuming a smooth, continuous and unimodal objective function are expected to run into difficulties if applied to optimizing $\mathcal{O}$ far from the global minimum.

In our work, the minimization of the objective function $\mathcal{O}$ is based on Particle Swarm Optimization (PSO) 4, which is known to be an effective and efficient computational method for solving other vision optimization problems (e.g. [16|9|24]). Canonical PSO, the simplest of PSO variants, has several attractive properties. Most importantly, it does not require knowledge of the derivatives of the objective function, depends on very few parameters, and requires a relatively low number of objective function evaluations until convergence [1. In our formulation, the rotation component of candidate poses is parameterized in terms of yaw, pitch, and roll angles, respectively. Translation is parameterized by its Euclidean coordinates.

\section{Experiments}

The accuracy benefits obtained using the proposed method are evaluated in this section. For brevity, FEATPOSE will refer to the feature-based pose estimation of Sec. 3.2 and TEXTPOSE to the proposed method. In all experiments, PSO employed 64 particles evolved through 64 generations. Search ranges were $\pm 20 \mathrm{~mm}$ for the translational parameters and $\pm 20^{\circ}$ for the rotational ones. These bounds could be tightened for faster PSO performance.

An experiment with synthetic images was conducted first, utilizing our renderer to accurately avail ground truth. A model of a densely textured rectangular cuboid of size $45 \times 45 \times 90 \mathrm{~mm}^{3}$ was rendered in 59 images $(1280 \times 960$ pixels, $22.2^{\circ} \times 16.7^{\circ} \mathrm{FOV}$ ) circumnavigating the object in a full circle perpendicular to its major symmetry axis and pointing at the object (see Fig. 22). The experiment was performed in three conditions, for circle radii of 500, 1000, and $1500 \mathrm{~mm}$. Using the ground truth data, we were able to calculate the error in the estimates. More specifically, denoting by $\mathbf{R}, \mathbf{t}$ the true object pose and by $\widehat{\mathbf{R}}, \widehat{\mathbf{t}}$ a pose estimate, the translational error is computed as $|\mathbf{t}-\widehat{\mathbf{t}}|$, whereas the rotational error is $\arccos \left(\left(\operatorname{trace}\left(\mathbf{R}^{-1} \widehat{\mathbf{R}}\right)-1\right) / 2\right)$ and corresponds to the amount of rotation about a unit vector that transfers $\mathbf{R}$ to $\widehat{\mathbf{R}}$. Table 1 summarizes the mean and standard deviation of the errors. TEXTPOSE improves the estimates of FEATPOSE, whereas all errors increase with the distance of the object from the camera, as expected. 

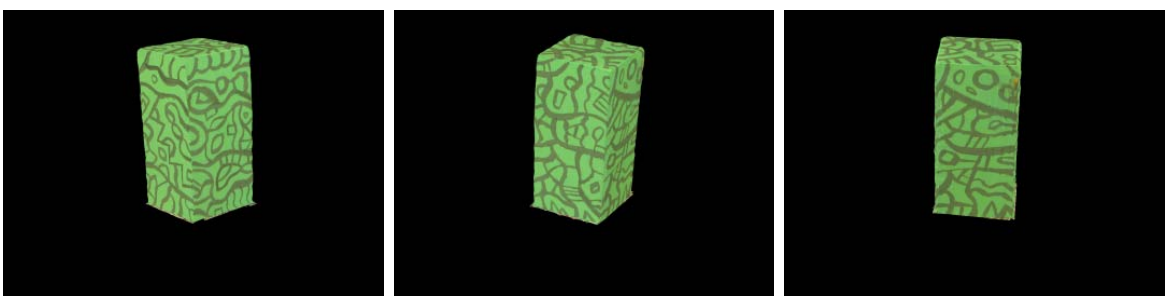

Fig. 2. Three of the synthetic images rendering the test object at $500 \mathrm{~mm}$

Table 1. Mean and standard deviation for the translational (t, $\mathrm{mm}$ ) and rotational $\left(\mathbf{R},{ }^{\circ}\right)$ pose errors for methods FEATpose and TEXTPOSE

\begin{tabular}{|c||c|c||c|c|}
\hline Radius & $\mathbf{t}_{\text {FEATPOSE }}$ & $\mathbf{R}_{\text {FEATPOSE }}$ & $\mathbf{t}_{\text {TEXTPOSE }}$ & $\mathbf{R}_{\text {TEXTPOSE }}$ \\
\hline $500 \mathrm{~mm}$ & $6.948(3.839)$ & $0.260(0.121)$ & $4.931(1.915)$ & $0.167(0.064)$ \\
\hline $1000 \mathrm{~mm}$ & $12.211(8.218)$ & $0.492(0.256)$ & $5.389(9.067)$ & $0.167(0.270)$ \\
\hline $1500 \mathrm{~mm}$ & $13.654(7.508)$ & $0.532(0.269)$ & $6.237(3.049)$ & $0.165(0.101)$ \\
\hline
\end{tabular}

The FEATPOSE and TEXTPOSE methods are compared next with the aid of real images of an object with sparser texture relative to the previous one. The object and the experimental setup are shown in Fig. 3. To obtain ground truth for object poses, a checkerboard was used to guide object laying, facilitating its placement on checkers and alignment with them. The object was placed upon every white checker of the $9 \times 10$ checkerboard. The checkerboard was at a distance of $\approx 0.75 \mathrm{~m}$ from the camera $\left(960 \times 1280\right.$ pixels, $\left.16^{\circ} \times 21^{\circ} \mathrm{FOV}\right)$ and each checker was $19 \times 19 \mathrm{~mm}^{2}$. As we lacked any information on the orientation of the intrinsic reference frame of the object, we only measured the translational error. The mean error in these 45 trials was $13.25 \mathrm{~mm}(2.19 \mathrm{~mm}$ std) for FEATPOSE and $8.3 \mathrm{~mm}$ (4.65 $\mathrm{mm}$ std) for TEXTPOse.

We also compared the methods with the aid of a more elaborate object, shown in Fig. 4. Due to its complex shape, ground truth regarding its pose was difficult to obtain. The initial pose provided by FEATPOSE was quite accurate owing
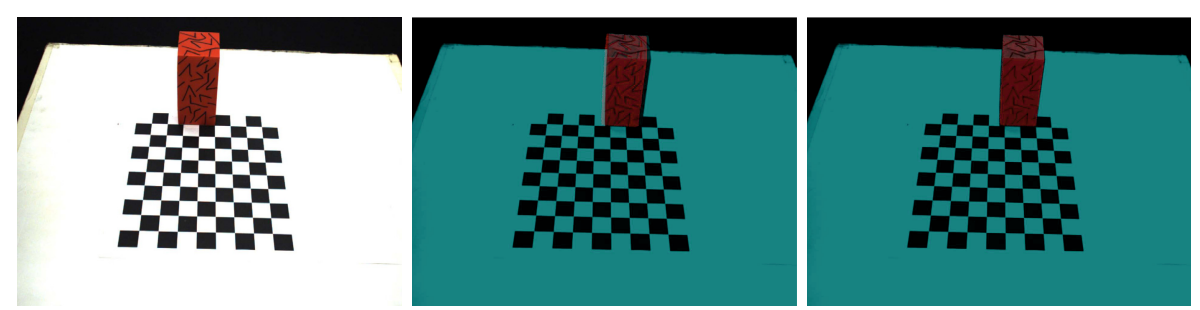

Fig. 3. Left to right: original image, FEATpose, and Textpose results. The original image is converted to grayscale and shown on the blue and green channels of an RGB color image. The red channel of each color image shows the estimate. The misalignment caused by an inaccurate FEATPOSE estimate is visible in the right vertical edge of the object in the middle image, whereas it has been corrected in the right image. 

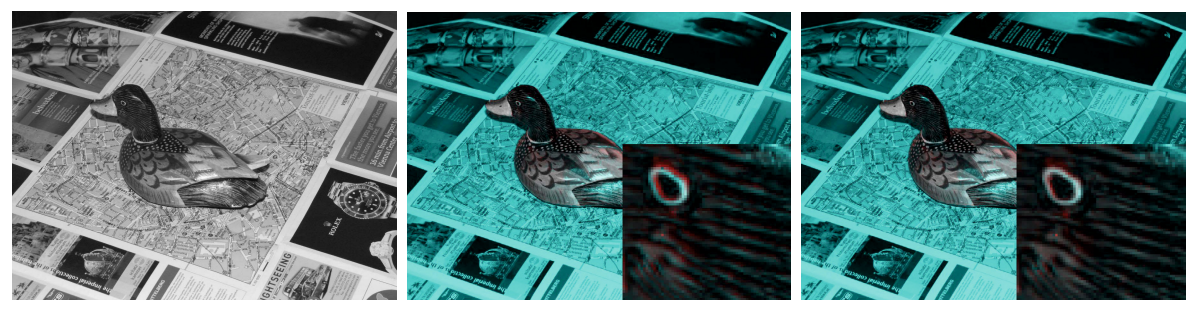

Fig. 4. Left to right: original image, FEATPOSE, and TEXTPOSE results with magnified detail near the eye, shown in the bottom-right. Results are encoded as in Fig. 3 .

to the very dense texture of the object, leaving little room for improvement. Nevertheless, the proposed method still managed to improve the pose estimate.

The execution time of the TEXTPOSE method is determined by the following factors. The number of pixels by which the model is rendered in the generated images increases linearly the complexity of the method. The complexity of the PSO algorithm is linear to the number of particles $P$ and generations $G$ considered. Finally, the number of triangles in the model also linearly increases computational complexity. Indicatively, when the method was executed for images of $960 \times 1280$ and a model of $5 \cdot 10^{4}$ triangles, execution time was $10 \mathrm{sec}$ on an Intel Core i7 CPU 950 @ 3.07GHz and a GeForce GTX 580 GPU.

The experiments confirm that the proposed method provides accurate refinements to the feature-based initial pose estimate. The method is most effective when the texture of the object of interest is weak (i.e. as in Fig. (3)), as in such cases the inaccuracy of the feature-based initialization is prone to be larger. Nevertheless, improvements are observed even for objects with dense texture.

\section{Conclusions}

This paper has presented an approach for rigid object pose estimation that was shown experimentally to be highly accurate and versatile. The approach combines feature and texture-based matching for pose estimation and is applicable to any kind of rigid free-form objects. Future work will address the extension of the method to multiple views and its application to the problem of continuous tracking of moving objects. Another direction concerns the tuning of PSO implementation and parameters in order to minimize execution time.

Acknowledgements. This work has received funding from the EC FP7 programme under grant no. 270138 DARWIN.

\section{References}

1. Angeline, P.: Evolutionary Optimization Versus Particle Swarm Optimization: Philosophy and Performance Differences. In: Porto, V.W., Waagen, D. (eds.) EP 1998. LNCS, vol. 1447, pp. 601-610. Springer, Heidelberg (1998)

2. Brox, T., Rosenhahn, B., Cremers, D., Seidel, H.-P.: High accuracy optical flow serves 3-D pose tracking: Exploiting contour and flow based constraints. In: Leonardis, A., Bischof, H., Pinz, A. (eds.) ECCV 2006. LNCS, vol. 3952, pp. 98111. Springer, Heidelberg (2006) 
3. Brox, T., Rosenhahn, B., Gall, J., Cremers, D.: Combined Region and MotionBased 3D Tracking of Rigid and Articulated Objects. PAMI 32(3), 402-415 (2010)

4. Eberhart, R., Shi, Y., Kennedy, J.: Swarm Intelligence. Morgan Kaufmann (2001)

5. Fischler, M., Bolles, R.: Random Sample Consensus: A Paradigm for Model Fitting with Applications to Image Analysis and Automated Cartography. CACM 24, 381395 (1981)

6. Gall, J., Rosenhahn, B., Seidel, H.P.: Robust Pose Estimation with 3D Textured Models. In: Chang, L.-W., Lie, W.-N. (eds.) PSIVT 2006. LNCS, vol. 4319, pp. 84-95. Springer, Heidelberg (2006)

7. Gall, J., Rosenhahn, B., Seidel, H.P.: Drift-Free Tracking of Rigid and Articulated Objects. In: Proc. of CVPR 2008, pp. 1-8 (2008)

8. Gordon, I., Lowe, D.G.: What and Where: 3D Object Recognition with Accurate Pose. In: Ponce, J., Hebert, M., Schmid, C., Zisserman, A. (eds.) Toward CategoryLevel Object Recognition. LNCS, vol. 4170, pp. 67-82. Springer, Heidelberg (2006)

9. Ivekovič, S., Trucco, E., Petillot, Y.: Human Body Pose Estimation with Particle Swarm Optimisation. Evolutionary Computation 16(4), 509-528 (2008)

10. Lepetit, V., Fua, P.: Monocular Model-Based 3D Tracking of Rigid Objects. Found. Trends. Comput. Graph. Vis. 1(1), 1-89 (2005)

11. Lourakis, M., Zabulis, X.: Accurate Scale Factor Estimation in 3D Reconstruction. In: Wilson, R., Hancock, E., Bors, A., Smith, W. (eds.) CAIP 2013, Part I. LNCS, vol. 8047, pp. 498-506. Springer, Heidelberg (2013)

12. Lourakis, M., Zabulis, X.: Model-Based Pose Estimation for Rigid Objects. In: Chen, M., Leibe, B., Neumann, B. (eds.) ICVS 2013. LNCS, vol. 7963, pp. 83-92. Springer, Heidelberg (2013)

13. Lowe, D.: Three-Dimensional Object Recognition from Single Two-Dimensional Images. Artificial Intelligence 31(3), 355-395 (1987)

14. Lowe, D.: Distinctive Image Features from Scale-Invariant Keypoints. Int. J. Comput. Vis. 60(2), 91-110 (2004)

15. Özuysal, M., Lepetit, V., Fleuret, F., Fua, P.: Feature Harvesting for Trackingby-Detection. In: Leonardis, A., Bischof, H., Pinz, A. (eds.) ECCV 2006. LNCS, vol. 3953, pp. 592-605. Springer, Heidelberg (2006)

16. Padeleris, P., Zabulis, X., Argyros, A.: Head Pose Estimation on Depth Data Based on Particle Swarm Optimization. In: CVPR Workshops, pp. 42-49 (2012)

17. Prisacariu, V., Reid, I.: PWP3D: Real-Time Segmentation and Tracking of 3D Objects. Int. J. Comput. Vis., 1-20 (2012)

18. Romea, A., Berenson, D., Srinivasa, S., Ferguson, D.: Object recognition and full pose registration from a single image for robotic manipulation. In: ICRA (2009)

19. Rothganger, F., Lazebnik, S., Schmid, C., Ponce, J.: 3D Object Modeling and Recognition Using Local Affine-Invariant Image Descriptors and Multi-View Spatial Constraints. Int. J. Comput. Vis. 66(3), 231-259 (2006)

20. Schmaltz, C., Rosenhahn, B., Brox, T., Weickert, J.: Region-Based Pose Tracking with Occlusions Using 3D Models. Mach. Vision Appl. 23(3), 557-577 (2012)

21. Snavely, N., Seitz, S., Szeliski, R.: Photo Tourism: Exploring Photo Collections in 3D. ACM Trans. Graph. 25(3), 835-846 (2006)

22. Vacchetti, L., Lepetit, V., Fua, P.: Stable Real-Time 3D Tracking Using Online and Offline Information. PAMI 26(10), 1385-1391 (2004)

23. Vergauwen, M., Gool, L.V.: Web-based 3D Reconstruction Service. Mach. Vision Appl. 17(6), 411-426 (2006)

24. Zhang, X., Hu, W., Maybank, S., Li, X., Zhu, M.: Sequential Particle Swarm Optimization for Visual Tracking. In: CVPR, pp. 1-8 (2008) 